\title{
CARACTERÍSTICAS CLÍNICAS Y EPIDEMIOLÓGICAS DE LOS PACIENTES VIH/SIDA DIAGNOSTICADOS CON TUBERCULOSIS EN EL SERVICIO DE ATENCIÓN INTEGRAL DEL HOSPITAL INFANTIL DR. ROBERT REID CABRAL DURANTE EL PERIODO 2010-2016
}

\section{Clinical and epidemiological characteristics of HIV/AIDS patients diagnosed with tuberculosis in the Integral Care Service of the Dr. Robert Reid Cabral Children's Hospital during the period 2010-2016}

\author{
Ricardo Elías-Melgena, Rosa Abreub, Milandres Garcíac
}

Recibido: 22 de abril, 2020 • Aprobado: 12 de junio, 2020

Cómo citar: Elías Melgen R, Abreu R, García M. Características clínicas y epidemiológicas de los pacientes VIH/SIDA diagnosticados con tuberculosis en el Servicio de Atención Integral del Hospital Infantil Dr. Robert Reid Cabral durante el periodo 2010-2016. cysa [Internet]. 17 de septiembre de 2020 [citado 18 de septiembre de 2020];4(3):123-129. Disponible en: https://revistas.intec.edu.do/ index.php/cisa/article/view/1908

\section{Resumen}

Introducción: el diagnóstico de TB puede ser particularmente difícil en niños infectados con VIH porque es común la superposición clínica con otra enfermedad relacionada con el VIH, lo que dificulta y retrasa un correcto diagnóstico.

Objetivo: conocer las características clínicas y epidemiológicas de los pacientes VIH/SIDA diagnosticados con tuberculosis en el Hospital Infantil Dr. Robert Reid Cabral durante el periodo 2010- 2016.

Métodos: se realizó un estudio descriptivo, la información se obtuvo de los expedientes de los pacientes VIH/SIDA diagnosticados con tuberculosis en el Hospital Infantil Dr. Robert Reid Cabral durante el periodo 2010-2016. Las variables de estudio se recolectaron en un instrumento diseñado para estos fines. Los datos se procesaron mediante el paquete estadístico SPSS 2007 y en el análisis se usó estadística descriptiva.

\footnotetext{
a Departamento de Neumología, Hospital Infantil Dr. Robert Reid Cabral, Centro Nacional de Investigación en Salud Materno Infantil Dr. Hugo Mendoza (CENISMI), República Dominicana.

Correo-e: reliasmelgen@yahoo.es ORCID: 0000-0001-7901-0532
}

\begin{abstract}
Introduction: The diagnosis of $\mathrm{TB}$ can be particularly difficult in children infected with HIV because clinical overlap with another HIV-related disease, making it difficult and delaying a correct diagnosis.

Objective: To know the clinical and epidemiological characteristics of HIV / AIDS patients diagnosed with Tuberculosis in Dr. Robert Reid Cabral Children's Hospital during the period 2010-2016.

Methods: Descriptive study, the information was obtained from the records of HIV / AIDS patients diagnosed with Tuberculosis in Dr. Robert Reid Cabral Children's Hospital during the period 2010-2016. The study variables were collected in a instrument designed for these purposes. The data were processed using the SPSS 2007 statistical package and the analysis was carried out using descriptive statistics.
\end{abstract}

\footnotetext{
b Servicio Atención Integral Dr. Teófilo Gautier Abreu, Hospital Infantil Dr. Robert Reid Cabral. Correo-e: rosaabreu82@hotmail.com

${ }^{c}$ Departamento de Neumología Hospital Infantil Dr. Robert Reid Cabral. Correo-e: milandresgarcia@hotmail.com
} 
Resultados: el $13.2 \%$ de los pacientes con VIH desarrollaron TB, $72.3 \%$ presentaba una inmunosupresión grave al momento del diagnóstico de TB, 45 \% refería el antecedente de contacto TB y el $90.5 \%$ era intradomiciliario. $53 \%$ no estaba recibiendo TARV antes de establecer el diagnóstico TB. Tos (65.9\%), fiebre $(61.7 \%)$ y pérdida o mal progreso de peso $(40.4 \%)$, fueron las principales manifestaciones clínicas. $76.6 \%$ completó 6 meses de tratamiento, $17 \%$ se perdió durante el seguimiento y $4.3 \%$ falleció durante el tratamiento.

Conclusión: $13.2 \%$ de los pacientes con VIH desarrollaron tuberculosis, con un importante deterioro de su inmunidad al momento de hacer este diagnóstico, más de la mitad no recibía terapia antiretroviral; un alto porcentaje de estos pacientes tenían antecedente de contacto intradomiciliario con un caso TB, $17 \%$ abandono tratamiento antituberculosis luego de iniciado.

Palabras clave: tuberculosis infantil; VIH/SIDA; coinfección TB/SIDA; diagnóstico TB/VIH; factores de riesgo TB/VIH.

\section{Introducción}

Las personas que viven con el virus de la inmunodeficiencia humana (VIH) tienen mayor probabilidad de enfermar por tuberculosis (TB) que las que viven sin el VIH; la TB es la principal enfermedad oportunista y la que mayor mortalidad produce entre las personas infectadas por el VIH. ${ }^{1}$

Las manifestaciones clínicas características de la TB tienen una forma de presentación diferente cuando existe inmunosupresión, siendo la manifestación pulmonar con baciloscopía negativa y la TB extrapulmonar más frecuentes en pacientes con el VIH que en aquellos sin VIH. Estas diferencias clínicas condicionan a un retraso en el diagnóstico de TB en las personas con VIH, relacionado este retraso con una elevada mortalidad. ${ }^{2}$

Los niños, principalmente aquellos menores de 5 años de edad, tienen de por sí un mayor riesgo de desarrollar enfermedad por TB luego de la infección por el Mycobacteria tuberculosis y este riesgo
Results: $13.2 \%$ of HIV patients developed TB, $72.3 \%$ had severe immunosuppression at the time of TB diagnosis, $45 \%$ reported a history of $\mathrm{TB}$ contact, and $90.5 \%$ were intradomiciliary. $53 \%$ were not receiving ART before establishing the TB diagnosis. Cough (65.9\%), fever $(61.7 \%)$ and weight loss or poor progress (40.4\%) were the main clinical manifestations. $76.6 \%$ completed 6 months of treatment, $17 \%$ was lost to follow-up, and $4.3 \%$ died during treatment.

Conclusion: $13.2 \%$ of HIV patients developed tuberculosis, with a significant deterioration in their immunity at the time of making this diagnosis, more than half did not receive antiretroviral therapy; a high percentage of these patients had a history of intradomiciliary contact with a TB case, $17 \%$ abandoned antituberculosis treatment after it was started.

Keywords: Childhood tuberculosis; HIV/AIDS; TB/AIDS co-infection; TB/HIV diagnosis; TB/HIV risk factors.

aumenta aún más en aquellos con VIH, relacionándose con el grado de inmunosupresión; los niños que viven con el VIH con una inmunidad grave tienen un riesgo cinco veces mayor de desarrollar enfermedad TB en comparación con niños con inmunosupresión leve. ${ }^{3}$

El diagnóstico de TB puede ser particularmente difícil en niños infectados con VIH, porque es común la superposición clínica con otra enfermedad relacionada con el VIH; por otro lado, la Terapia Antirretroviral (TARV) reduce significativamente el riesgo de que los niños con VIH desarrollen $\mathrm{TB}$, pero solo la mitad de los niños elegibles, acceden a esta TARV. ${ }^{4}$

En República Dominicana, tanto el VIH, la TB y la coinfección TB/VIH representan un importante problema de salud, la proporción de casos TB/VIH con relación al total de casos diagnosticados de TB ha aumentado significativamente de $5.1 \%$ en el año 2000 a $9 \%$ en el año $2009^{5}$ y actualmente representan el $25 \%$ de los casos nuevos por TB, lo 
cual se considera una muy alta carga. ${ }^{6}$ En una serie de 249 casos diagnosticados con TB en el Hospital Infantil Dr. Robert Reid Cabral (HIRRC) durante el periodo 2007-2012, el $6 \%$ tenía coinfección TB/VIH. ${ }^{7}$

El objetivo de la presente investigación es conocer las características clínicas y epidemiológicas de los pacientes VIH/SIDA diagnosticados con tuberculosis en el Servicio de Atención Integral del Hospital Infantil Dr. Robert Reid Cabral durante el periodo 2010-2016.

\section{Material y método}

Estudio descriptivo para conocer las características clínicas y epidemiológicas de los pacientes VIH/ SIDA diagnosticados con TB en el Servicio de Atención Integral (SAI) del HIRRC durante el periodo 2010-2016, la información se obtuvo de los expedientes clínicos de los pacientes VIH/SIDA diagnosticados con TB en el SAI del HIRRC durante el periodo 2010-2016, las variables de estudio se recolectaron en un instrumento diseńado para estos fines. Los datos se procesaron mediante el paquete estadístico SPSS 2007 y para el análisis se usó estadística descriptiva. Toda la información recolectada se utilizó bajo estricta confidencialidad por parte de los investigadores, y no se dio a conocer datos pertinentes de los pacientes y sus familiares.

\section{Resultados}

Durante los siete años del periodo estudiado, en el SAI del HIRRC se evaluaron 357 pacientes con VIH, el $13.2 \%$ (47) de estos pacientes desarrollaron TB. El $91.4 \%$ (43) de los pacientes con la coinfección TB/VIH TB había adquirido la infección por $\mathrm{VIH}$ de forma vertical. Al momento de ser diagnosticado con TB, el $87.2 \%$ (41) de estos pacientes tenía una clasificación clínica categoría $\mathrm{C}$ y el $72.3 \%$ (34) presentaba una inmunosupresión grave, con menos del $15 \%$ de linfocito CD 4 en el $63.8 \%$ (30) de los casos y el $59.6 \%$ (28) con una carga viral de más de 100,000 copias (tabla 1).

Tabla 1. Estado inmunológico de los pacientes VIH que desarrollaron $\mathrm{TB}$

\begin{tabular}{|c|c|c|}
\hline Vía transmisión & Frecuencia & $\%$ \\
\hline Transmisión vertical & 43 & 91.4 \\
\hline Transfusión sanguínea & 1 & 2.2 \\
\hline Indeterminada & 3 & 6.4 \\
\hline \multicolumn{3}{|l|}{ categoría clínica } \\
\hline Categoría A & 1 & 2.2 \\
\hline Categoría B & 5 & 10.6 \\
\hline Categoría C & 41 & 87.2 \\
\hline \multicolumn{3}{|l|}{$\begin{array}{l}\text { Grado de supresión } \\
\text { Inmunológica }\end{array}$} \\
\hline $\begin{array}{l}\text { Sin evidencia de } \\
\text { inmunosupresión }\end{array}$ & 1 & 2.2 \\
\hline Supresión moderada & 12 & 25.5 \\
\hline Supresión grave & 34 & 72.3 \\
\hline \multicolumn{3}{|l|}{ Porcentaje CD 4} \\
\hline Menos 15\% & 30 & 63.8 \\
\hline $16-25 \%$ & 15 & 31.9 \\
\hline Mayor 25\% & 2 & 4.3 \\
\hline \multicolumn{3}{|l|}{ Carga Viral } \\
\hline Indetectable & 2 & 4.3 \\
\hline menos de 50,000 copias & 10 & 21.2 \\
\hline 50,000-99,999 copias & 7 & 14.9 \\
\hline Más de 100,000 copias & 28 & 59.6 \\
\hline
\end{tabular}

Fuente: expedientes clínicos SAI HIRRC.

En el $36.2 \%$ de los casos, el diagnóstico de TB se realizó cuando el paciente tenía entre 1-5 años de edad y en el 51.1\% entre los 6-15 ańos de edad (tabla 2). 
Tabla 2. Edad de los pacientes VIH al ser diagnosticado TB

\begin{tabular}{|c|c|c|c|}
\hline $\begin{array}{c}\text { Edad al } \\
\text { diagnóstico de } \\
\text { TB }\end{array}$ & Frecuencia & $\mathbf{\%}$ & $\begin{array}{c}\text { \% } \\
\text { acumulado }\end{array}$ \\
\hline menor 1 año & 4 & 8.5 & 8.5 \\
\hline 1-5 años & 17 & 36.2 & 44.7 \\
\hline 6-15 años & 24 & 51.1 & 95.8 \\
\hline más de 15 años & 2 & 4.2 & 100 \\
\hline Total & $\mathbf{4 7}$ & $\mathbf{1 0 0}$ & \\
\hline
\end{tabular}

Fuente: expedientes clínicos SAI HIRRC.

El $60 \%$ (28) de estos pacientes vivían en hacinamiento, más de 3 personas por habitación y el 45 $\%$ (21) refería el antecedente de haber estado en contacto con una persona enferma por TB, siendo este contacto en el $90.5 \%$ (19) de los casos, un contacto intradomiciliario (tabla 3 ).

Tabla 3. Factores de riesgo para desarrollar TB

\begin{tabular}{|l|c|c|}
\hline Factor de riesgo & Frecuencia & \% \\
\hline Hacinamiento & 28 & 60 \\
\hline Antecedentes contacto TB & 21 & 45 \\
\hline $\begin{array}{l}\text { Contacto intradomici- } \\
\text { liario }\end{array}$ & 19 & 90.5 \\
\hline
\end{tabular}

Fuente: expedientes clínicos SAI HIRRC.

En la tabla 4, se muestra que el $53 \%$ (25) de los pacientes, no estaba recibiendo TARV antes de establecer el diagnóstico TB, el $6 \%$ (3) de estos la había abandonado y $47 \%$ (22) nunca habían recibido TARV.
Tabla 4. TARV en los pacientes antes del diagnóstico de TB

\begin{tabular}{|l|c|c|}
\hline \multicolumn{1}{|c|}{ TARV } & Frecuencia & \% \\
\hline Con TARV & 22 & 47 \\
\hline Sin TARV & 22 & 47 \\
\hline Abandono TARV & 3 & 6 \\
\hline
\end{tabular}

Fuente: expedientes clínicos SAI HIRRC.

La tos seca o productiva, en el $65.9 \%$ de los casos, fiebre en el $61.7 \%$, la desnutrición $60 \%$ y pérdida o mal progreso de peso en el $40.4 \%$, fueron las principales manifestaciones clínicas en los pacientes con VIH diagnosticados TB (tabla 5).

Tabla 5. Manifestaciones clínicas de los pacientes

\begin{tabular}{|l|c|}
\hline \multicolumn{1}{|c|}{ Manifestaciones clínicas } & $\%$ \\
\hline Tos (seca o productiva) & 65.9 \\
\hline Fiebre & 61.7 \\
\hline Desnutrición & 64.0 \\
\hline Pérdida o mal progreso de peso & 40.4 \\
\hline Sudoración & 6.4 \\
\hline Irritabilidad & 2.1 \\
\hline Otros menos frecuentes & 23.4 \\
\hline
\end{tabular}

Fuente: expedientes clínicos SAI HIRRC.

El resultado al tratamiento antituberculosis de estos pacientes muestra que el $76.6 \%$ (36) completó durante 6 meses el tratamiento con mejoría de su cuadro clínico (éxito al tratamiento), el $17 \%$ (8) no completó tratamiento y se perdió durante el seguimiento, el $4.3 \%$ (2) falleció durante el tratamiento y un paciente no mejoró su sintomatología (fracaso) durante el tratamiento (tabla 6). 
Tabla 6. Resultado al tratamiento antituberculosis

\begin{tabular}{|l|c|c|}
\hline \multicolumn{1}{|c|}{ Resultado } & Frecuencia & $\%$ \\
\hline Éxito al tratamiento & 36 & 76.6 \\
\hline Fracaso & 1 & 2.1 \\
\hline Fallecidos & 2 & 4.3 \\
\hline Perdido en el seguimiento & 8 & 17 \\
\hline \multicolumn{1}{|c|}{ Total } & $\mathbf{4 7}$ & $\mathbf{1 0 0}$ \\
\hline
\end{tabular}

Fuente: expedientes clínicos SAI HIRRC.

\section{Discusión}

El 13.2\% de los pacientes con VIH que se evaluaron durante el periodo de estudio desarrollaron TB; siendo esta la principal causa de enfermedad y muerte en personas infectadas por el VIH. ${ }^{8}$ Luego de producirse la infección por el Mycobacterium tuberculosis, el riesgo de desarrollar la enfermedad por TB es mayor en menores de 5 años de edad y en personas que viven con el $\mathrm{VIH} .^{9-11}$

Un aumento en la carga viral del VIH en los pacientes produce una disminución de los linfocitos T CD4+ y ocasiona una depresión en la respuesta del sistema inmunitario, condicionando la aparición de enfermedades oportunista como la TB; la Terapia Antirretroviral ha cambiado drásticamente la historia natural de la infección por el VIH, ya que se consigue una disminución prolongada de la carga viral. ${ }^{12}$

Al momento de ser diagnosticado TB, el $72.3 \%$ de los pacientes evaluados mostraban un grado de inmunosupresión grave, con una carga viral sobre 100,000 copias, $63 \%$ presentaban menos de un $15 \%$ de linfocitos CD4 y el $53 \%$ de los pacientes no estaban recibiendo terapia antiretroviral al momento de diagnosticarse la enfermedad por TB.

El 91.4\% (43) de los pacientes habían adquirido la infección por VIH de forma vertical y el diagnóstico de TB se había hecho en el $36.2 \%$ de los casos cuando el paciente tenía entre 12-60 meses de edad y en el 51.1\% entre los 72-180 meses de edad. En una casuística de 246 niños que adquirieran el VIH por transmisión vertical, solo el 2,4\% desarrollaron $\mathrm{TB}$, estos pacientes al momento del diagnóstico de TB se encontraban en las edades entre 4 y 160 meses (promedio 40,8 meses). ${ }^{13}$

Otros factores de riesgo para desarrollar TB en estos pacientes, además del grado de inmunosupresión que presentaban, es el hecho de que el $60 \%$ vivía en hacinamiento, y el $45 \%$ refería el antecedente de haber estado en contacto con una persona enferma por TB, siendo este contacto en el $90.5 \%$ de los casos, un contacto intradomiciliario.

En los niños es siempre difícil el diagnóstico de TB, esto se ve agravado más aún cuando coexisten la TB y la infección por VIH, ya que la sintomatología se hace atípica en la medida que disminuye la inmunidad. En los niños infectados con VIH se debe sospechar TB ante la presencia persistente de fiebre, tos y taquipnea, adenopatías periféricas, pérdida de peso superior al $10 \%$ y también cuando hay adultos en la casa que son tosedores crónicos. ${ }^{14}$

La tos seca o productiva (65.9\%), fiebre (61.7\%), desnutrición $(60 \%)$ con pérdida o mal progreso de peso $(40.4 \%)$ fueron las principales manifestaciones clínicas en los pacientes con VIH diagnosticados TB en nuestro estudio.

El esquema de elección para el tratamiento anti-TB en niños con VIH de cualquier forma de TB drogo sensible, es un esquema diario durante 6 meses, excepto en los casos de TB ósea, TB miliar y meningitis TB, que debe ser durante 12 meses. $^{2}$

En el resultado del tratamiento de los pacientes evaluados, vemos que el 76.6 \% completó el tratamiento anti tuberculosis, con mejoría de las manifestaciones clínica y radiológicas y el porcentaje de fallecidos fue de $4.3 \%$, hay que hacer notar que el $17 \%$ no completó el tratamiento, perdiéndose durante el seguimiento. 
En una investigación realizada para medir la tasa de cumplimiento y abandono al tratamiento antirretroviral en personas seropositivas al VIH en la República Dominicana, se reportó que el 30.5\% de los pacientes con VIH no cumplió con la cita programada para retirar el tratamiento antirretroviral y el $13.2 \%$ reportó haber dejado en algún momento de tomar los medicamentos. ${ }^{15}$

Según el informe de gestión de la División de TB del Ministerio de Salud, en donde se reportan los resultados al tratamiento anti tuberculosis a nivel nacional, el $19 \%$ de los casos TB/VIH en tratamiento anti tuberculosis se perdieron durante el seguimiento, y otro $19 \%$ falleció. ${ }^{16}$

En conclusión, la probabilidad de desarrollar enfermedad por TB en los niños infectados por VIH es sumamente elevada; las manifestaciones clínicas de la TB están muy influenciadas por el grado de inmunosupresión que puedan tener estos pacientes, dificultando aún más el diagnóstico.

Todo niño con VIH, con signos y síntomas sugestivos de TB como son la tos, pérdida de peso o retraso en el crecimiento y con el antecedente de contacto de un caso de TB debe ser investigado para TB. Una vez establecido el diagnóstico de TB activa, se debe iniciar de inmediato tratamiento anti-TB. La terapia antirretroviral debe empezar tan pronto como se compruebe la tolerancia al tratamiento anti-TB, vigilando siempre el riesgo de toxicidad y efectos secundarios.

\section{Bibliografía}

1. Unión Internacional de Lucha Contra la Tuberculosis (La Unión). Manejo de la Tuberculosis. Una Guía Esencial de Buenas Prácticas. [Internet]. (6ta. ed.); 2010. [Revisado el 26 de abril 2020]. Disponible en http://files. sld.cu/sida/files/2016/06/manejo-de-la-tuberculosis.pdf
2. Organización Panamericana de la Salud. Coinfección TB/VIH. Guía Clínica Regional. [Internet]. [Actualización 2017. Revisado el 26 de abril 2020]. Disponible en http://www1. paho.org/hq/dmdocuments/2011/Coinfeccion_TB-VIH_Guia_Clinica_TB.pdf

3. Organización Mundial de la Salud. Guidance for national tuberculosis programmes on the management of tuberculosis in children [Internet]. ( $2^{\text {nd }}$ ed.); 2014. [Revisado el 26 de abril 2020]. Disponible en http://apps. who.int/medicinedocs/documents/s21535en/ s21535en.pdf

4. Organización Mundial de la Salud. Roadmap towards ending TB in children and adolescents. [Internet]; 2018. [Revisado el 26 de abril 2020]. Disponible en http://apps.who.int/iris/ bitstream/handle/10665/274374/978924151 4668-eng.pdf?ua $=1$

5. ElíasMelgen, R.Coinfección porelVirusdeInmunodeficiencia Humana y Tuberculosis en República Dominicana. Análisis de tendencias. Boletín CENISMI. sept-dic 2010;20(3):1-12 Disponible en https://www.ministeriodesalud.go.cr/ index.php/investigacion-y-tecnologia-en-salud/ inventarios/inventario-blibiografico-vih-tb/ investigacion-vih-sida-tb/1446-coinfeccion-por-vih-y-tuberculosis-en-republica-dominicana-analisis-de-tendencias-1/file

6. Organización Mundial de la Salud. Global tuberculosis report 2018. Tuberculosis country profiles. [Internet]. Disponible en https:// extranet.who.int/sree/Reports?op=Replet $\&$ name $=\% 2 \mathrm{FWHO} H \mathrm{HQ}$ _Reports $\% 2 \mathrm{FG} 2 \%$ 2FPROD\%2FEXT\%2FTBCountryProfile\&I$\mathrm{SO} 2=\mathrm{DO} \& \mathrm{LAN}=\mathrm{ES} \&$ outtype $=$ pdf [Revisado el 26 de abril 2020].

7. Elias Melgen R, Pantaleón E C, Lazala J A, Abreu B. A. Características clínico epidemiológicas de 
los casos de tuberculosis infantil diagnosticados en el Hospital Infantil Dr. Robert Reid Cabral 2007- 2012. Ciencia y Salud, 2019;3(1):9-13. Disponible en https://revistas.intec.edu.do/ index.php/cisa/article/view/1325

8. Lozano Salazar JL. Coinfección por tuberculosis y virus de la inmunodeficiencia humana: confluencia de dos epidemias. Medisan 2012;16(9):1438. Disponible en http://scielo.sld.cu/scielo.php?script $=$ sci_ arttext\&pid=S1029-30192012000900015

9. Hesseling C, SchaafHS, Gie RP, Starke JR, Beyers $\mathrm{N}$. A critical review of diagnostic approaches used in the diagnosis of childhood tuberculosis. Int J Tuberc Lung Dis 2002;6(12):1038-45. Disponible en http://docserver.ingentaconnect. com/deliver/connect/iuatld/10273719/v6n12/ s3.pdf?expires $=1556458887 \&$ id $=0000 \&$ titlei$\mathrm{d}=3764 \&$ checksum $=22742 \mathrm{~B} 0 \mathrm{D} 71 \mathrm{CAB} 0 \mathrm{~A}$ DC1C584D5EFBED532

10. Marais BJ, Gie RP, Schaaf HS, Hesseling AC, Obihara CC, Starke JJ, Enarson DA, Donald PR, Beyers N. The natural history of childhood intra-thoracic tuberculosis: a critical review of literature from the pre-chemotherapy era Int J Tuberc Lung Dis 2004;8(4):392-402. Disponible en http://docserver.ingentaconnect.com/deliver/connect/iuatld/10273719/ v8n 4/s2.pdf? expires $=1556460041 \& \mathrm{i}$ $\mathrm{d}=0000 \&$ titleid $=3764 \&$ checksum $=7448 \mathrm{D}$ 061714714524237DE994BD7F4A8

11. Organización Mundial de la Salud. La carga mundial de tuberculosis y su relación con el VIH. [Internet]. Disponible en https://www. who.int/tb/challenges/hiv/talking_points/es/ [Revisado el 26 de abril 2020].
12. Hernández Requejo D, Pérez Ávila J, Carr Pérez CA. Linfocitos TCD 4+ y carga viral en pacientes con debut de sida que reciben tratamiento antirretroviral. Revista Cubana de Investigaciones Biomédicas. 2014;33(3):304-12. Disponible en http://scielo.sld.cu/scielo.php?script=sci_arttex$\mathrm{t} \&$ pid=S0864-03002014000300006

13. Villarroel J, et al. Tuberculosis y SIDA en Pediatría: A propósito de cinco casos. Rev Chil Infect 2007;24(6):472-6. Disponible en http://dx.doi. org/10.4067/S0716-10182007000600007 https://scielo.conicyt.cl/scielo.php?script=sci_ arttext\&pid=S0716-10182007000600007

14. Villarroel J. Tuberculosis y SIDA en el niño. Neumología Pediátrica, 2008;3(2):165-70. Disponible en http://www.neumologia-pediatrica.cl/wp-content/uploads/2017/06/200832. pdf\#page $=24$

15. Pérez Then, E. Tasa de cumplimiento y abandono al tratamiento antirretroviral en personas seropositivas al VIH en la República Dominicana. CENISMI, serie de publicaciones técnica III; 2008.

16. Ministerio de Salud. Situación del control de la tuberculosis en la República Dominicana-2018. Informe de Gestión. República Dominicana; 2019 